\title{
La difusión del conocimiento contrahegemónico en Venezuela: el reto de las revistas científicas
}

\author{
Ochoa Henríquez, Haydée* \\ Boscán, Elizabeth Carolina**
}

\section{Resumen}

En el marco de un proceso de transformación que busca construir un sistema contrahegemónico al capitalismo, se producen en Venezuela desde fines de los noventa cambios significativos en casi todas las dimensiones de la vida, como parte de los cuales se promueve la producción de conocimiento alternativo al hegemónico alineado a la llamada corriente principal de la ciencia, el cual predomina en la mayoría de las revistas editadas en el país. Este trabajo tiene como propósito explorar la necesidad que las revistas científicas del país se abran a la publicación de conocimiento contrahegemónico. Los resultados revelan que en el contexto de una dominación de la corriente principal de la ciencia en las revistas latinoamericanas, de lo cual no escapa Venezuela, el gobierno promueve la producción de conocimiento contrahegemónico con poca presencia en las revistas del país, lo cual ha estado acompañado de políticas públicas incrementales que no impactan la necesidad de revertir esta situación, por el contrario recientemente tiende a formularse una política pública para las revistas nacionales que reproduce la corriente principal de la ciencia. Se concluye sobre la existencia en el país de una contradicción entre la política pública de producción de conocimiento contrahegemónico, el cual se encuentra marginado en las revistas científicas, y una política estatal de difusión con cambios incrementales que no impactan la transformación y en puerta proyectos que reproducen la vieja política.

Palabras clave: Corriente principal de la ciencia; conocimiento contrahegemónico; revistas científicas; Venezuela.

\section{Recibido: 10-05-16. Aceptado: 29-06-16}

* $\quad$ Dra. en Estudios del Desarrollo. Investigadora del Centro de Estudios de la Empresa de la Universidad del Zulia en el área de Gestión Pública. Acreditada en el PEII-ONCTI como Investigadora Emérita Nivel C. e-mail: haydeeochoa@gmail.com

** Magíster en Gerencia Pública. Estudiante del Doctorado en Ciencias para el Desarrollo Estratégico de la Universidad Bolivariana de Venezuela (UBV). Investigadora del Centro de Estudios de la Empresa de la Universidad del Zulia en el área de Gestión Pública. Acreditada en el PEIIONCTI como Investigadora B. e-mail: elizabeth.boscanap@gmail.com 


\section{The Spreading of Counter-Hegemonic Knowledge in Venezuela: The Challenge of Scientific Journals}

As part of a transformation process that seeks to build a counter-system to capitalism; in Venezuela, since the late nineties, significant changes are produced in almost all aspects of life, as part of which the production of alternative knowledge is promoted to hegemonic aligned with so called mainstream science, which predominates in most magazines published in the country. This paper aims to explore the need for scientific journals in the country to open up to the publication of counterhegemonic knowledge. The results show that in the context of a domination of the mainstream science in Latin American journals, which does not escape from Venezuela's reality, the government promotes the production of anti-hegemonic knowledge with little presence in magazines in this country; this has been accompanied by incremental public policies that do not impact the need to reverse this situation, on the contrary recently it tends to be formulated a public policy for national magazines that reproduces the mainstream science. We conclude the existence, in the country, of a contradiction between the public policy of production of counter-hegemonic knowledge, which is marginalized in the scientific magazines and a state policy diffusion with incremental changes that do not impact the transformation and projects that reproduce the old policy.

Keywords: Mainstream science; counter-hegemonic knowledge; scientific journals; Venezuela.

"Hay científicos cuya sensibilidad política los lleva a rechazar el sistema social reinante en nuestro país y en toda Latinoamérica... No aceptan sus normas y valores-copiados servilmente para colmo de valores extranjeros-; no aceptan el papel que el sistema les asigna, de ciegos proveedores de instrumentos para uso de cualquiera que pueda pagarlos y hasta sospechan de la pureza y neutralidad y apoliticismo de las élites científicas internacionales al imponer temas, métodos y criterios de evaluación" (Varsavsky, 1969)

\section{Introducción}

Varios países de América Latina, entre los cuales se encuentra Venezuela, viven desde fines de los noventa un proceso de búsqueda de un modelo de sociedad alternativo al capitalismo, se aspira un viraje del favorecimiento al capital, según las políticas de este país, a la construcción de: 1) Un Estado social y de justi- cia como lo indican los principios de la Constitución de la República Bolivariana de Venezuela (CRBV) (ANC, 1999), 2) La suprema felicidad social, como lo dice en Primer Plan Socialista de Venezuela (Presidencia de la República, 2007), 3) El favorecimiento de las grandes mayorías sociales como se expresa en las numerosas políticas públicas formuladas desde 1999 y 4) Un país contrahegemónico al 
capital como lo indican los cinco grandes objetivos estratégicos del Plan 2013$2019^{1}$ (AN, 2013).

En este contexto y en una lucha permanente con el modelo que se busca superar, tienen lugar transformaciones en todas las dimensiones de la vida, con diversas dinámicas de avances y retrocesos, de las cuales no ha escapado la realidad científico-técnica del país y como parte de ella los procesos y medios de difusión del conocimiento científico que se produce. El objetivo de este trabajo es explorar las condiciones en las cuales se encuentran las publicaciones científicas a la luz de la necesidad de difundir conocimiento alternativo a la corriente principal de la ciencia, también llamada por Varsavsky (2006) "ciencia universal", conocimiento alternativo que emerge en el marco de las transformaciones que se producen en el país.

\section{La corriente principal de la ciencia en las revistas científicas Latinoamericanas}

La mayoría de las revistas científicas latinoamericanas han asumido un perfil alineado a las exigencias de la lla- mada corriente principal de la ciencia, impuesta por el Science Citation Index (SCl) y más recientemente SCOPUS y Google entre otros.

El conocimiento de la corriente principal de la ciencia es un "conocimiento con pretensión universal y neutral presente en todas las disciplinas científicas, alineado a los temas e interrogantes de investigación surgidos desde centros hegemónicos de conocimiento" (Boscán, 2016).

En términos de Guedón (2011), el conocimiento que sustenta la corriente principal de la ciencia, se ha producido mediante una "estructura de poder en la ciencia" configurada en el proceso de difusión de conocimientos por los índices internacionales, especialmente el SCl. Siguiendo al autor "una de las principales funciones del $\mathrm{SCl}$ es adjudicar el orden jerárquico de las revistas de tal manera que se preserve la presente estructura "cartelizada» de publicaciones" (Guedón, 2011: 156).

En este contexto las revistas tienden a excluir conocimiento alternativo al dominante, el cual tiene poca presencia en estos medios de difusión de la ciencia. Esta situación se ha visto favorecida en el

Los cinco grandes objetivos estratégicos de este Plan son: 1) "Defender, expandir y consolidad el bien más preciado que hemos reconquistado después de 200 años: La independencia nacional", 2) "Continuar construyendo el socialismo bolivariano del siglo XXI en Venezuela como alternativa al sistema destructivo y salvaje del capitalismo y con ello asegurar la mayor suma de seguridad social, mayor suma de estabilidad política y la mayor suma de felicidad para nuestro pueblo", 3) "Convertir a Venezuela en un país potencia en lo social lo económico y lo político dentro de la gran potencia naciente de América Latina y el Caribe, que garanticen la conformación de una zona de paz en nuestra América", 4) "Contribuir al desarrollo de una nueva geopolítica internacional en la cual tome cuerpo un mundo multicéntrico y pluripolar que permita lograr el equilibrio del universo y garantizar la paz planetaria" y 5) "Contribuir a la preservación de la vida en el planeta y la salvación de la especie humana" (AN, 2013). 
contexto neoliberal en América Latina desde los años 90 , por los sistemas de promoción de la investigación, los cuales exigen a sus investigadores producción científica, publicada en revistas reconocidas por el SCl, cuestión que impulsó la creación de nuevas revistas que desde sus inicios se programaron para ingresar al referido índice, asumiendo para ello los criterios de aceptación del SCI. Destacan en esta lógica los sistemas de promoción de la investigación de México, Argentina y Venezuela, producto de una política asumida por muchos países en América Latina, bajo el impulso de los organismos multilaterales, en particular del Banco Mundial y el BID, que contribuyó a la reproducción del esquema de poder de organización de la producción científica.

A esto se agrega la creación de índices latinoamericanos alineados a los referidos criterios entre los cuales se destacan SCIELO, REDALYC y otros menos conocidos pero que igualmente cumplen un papel reproductor del conocimiento hegemónico. Estamos en presencia de bases de datos latinoamericanas que reproducen la llamada corriente principal de la ciencia.

Desde la lógica de reproducción del conocimiento dominante SCIELO y REDALYC, dado su acceso libre, cumplen un papel relevante. Según directivos de REDALYC la "profesionalización de la práctica científica" en nuestros países se asocia al incremento de "la cantidad de artículos publicados, y publicados, además, en revistas científicas de corriente principal" (Aguado y Becerril, 2016:12), postura comprometida con el mantenimiento de la estructura dominante de las publicaciones científicas y la subordina- ción a los temas e intereses de investigación exógenos.

La condición de acceso libre de los referidos índices, ha favorecido la difusión de la producción intelectual de la región y la comunicación entre la comunidad científica lo cual podría ser interpretado como favorable a la visibilidad de la ciencia latinoamericana, pero desde otra lectura este favorecimiento intensifica la reproducción de la corriente principal de la ciencia en la región. El acceso libre de estos índices requiere, para revertir la reproducción de la corriente principal de la ciencia, políticas que promuevan la difusión de conocimiento contrahegemónico con lo cual se favorece la democratización del conocimiento producido en la región.

En ciencias sociales se privilegia, como dice Lander (2000:62), conocimiento que asume "la sociedad de mercado como el único orden social posible. Se niega la posibilidad de imaginar modalidades de vida colectiva que no estén organizadas por la lógica del mercado".

En las llamadas ciencias duras la corriente principal promueve la biotecnología y la nanotecnología. Según Lander (2009:94) ambas son producto de un modo de conocimiento que tiene como objetivo "la superación de los límites tecnológicos y/o de costos para avanzar en el procesos de mercantilización" a través de "procesos científico-tecnológicos de manipulación/apropiación de la vida y de la materia mediante los cuales se han dado pasos colosales en el control sobre de las capacidades generativas de la vida para su conversión en mercancía".

La reproducción de la corriente principal de la ciencia en nuestras revis- 
tas entonces, tributa al control de la vida y la naturaleza por intereses privados para el mantenimiento de la sociedad capitalista cuyo eje son los países hegemónicos, cuestión oculta bajo el ropaje de neutralidad de ese tipo de conocimiento.

\section{El conocimiento contrahegemónico en las revistas científicas latinoamericanas}

El conocimiento contrahegemónico es un tipo de conocimiento enfrentado con el producido por la corriente principal de la ciencia. En términos sustantivos, el conocimiento contrahegemónico devela los límites del conocimiento de la corriente principal o "de punta" para la comprensión profunda de la realidades latinoamericanas, toma expresión en temas, métodos e interrogantes de investigación que desde el pensamiento crítico (marxismo, descolonialidad, pensamiento crítico latinoamericano) exponen la diversidad del conocimiento existente, la crisis del pensamiento eurocéntrico y el modelo de sociedad al cual tributa.

El conocimiento alternativo se produce en oposición al conocimiento dominante, así la visibilidad del conocimiento alternativo es eminentemente un tema político que supone interpelar al modo de difusión dominante. Según Guedón (2011:139:) "Las revistas científicas no son sólo órganos de difusión sino que también funcionan como espacios reguladores del acceso a la información" el proceso de edición de revistas conlleva al ejercicio de poder, en el cual el acceso al conocimiento alternativo es bloqueado, a nuestro juicio, por diversas vías.
El conocimiento que enfrenta la corriente principal generalmente no se cita en artículos del pensamiento hegemóni$\mathrm{co}$, ni siquiera por razones metodológicas de confrontación, no obstante se incorpora ocasionalmente en artículos comprometidos con la corriente principal pero de modo sesgado con uso interesado para avalar el pensamiento conservador. A modo de ejemplo, para Aguado y Becerril (2016:26) la pérdida de liderazgo científico en Venezuela se explica por condiciones internas para lo cual cita a Iraida Vargas poniendo en su discurso fuga de investigadores, ocultando el discurso contrahegemónico de Vargas (2012:2), quien en defensa de la necesidad de construir una "Ciencia Nuestra" pone sobre el tapete que la pérdida de talento joven de nuestras universidades se produce "porque la formación que les ofrecen las universidades se ha realizado de acuerdo a las normas que las metrópolis imponen" y "se ha agravado en la última década en la medida que las políticas mediáticas y culturales del imperio profundizan la desnacionalización de las conciencias de muchos jóvenes", se cita un autor ocultando su razonamiento contrahegemónico sobre la pérdida de talento en nuestro país. Esta estrategia de citas contribuye a la legitimación de los autores en tanto luce como disposición a la incorporación de pensamiento diverso.

El pensamiento contrahegemónico es excluido por las revistas a través de diversas vías, algunas imperceptibles:

1. Con frecuencia en trabajos de las ciencias sociales las revistas argumentan abiertamente politización y en consecuencia falta de objetividad y cientificidad, ocultando el papel ideológico del 
conocimiento desde cualquier perspecti$v^{2}{ }^{2}$. Según Varsavsky (1969:6) “Un cientificista no puede aceptar ocuparse de problemas relacionados con la política porque esa no es una actividad científica legítima según las normas de quienes desde el hemisferio Norte orientan las actitudes y opiniones de nuestros investigadores y sancionan virtudes y pecados". Los trabajos que critican los impactos del capital son vistos como trabajos ideológicos, mientras que los artículos que realizan descripciones que favorecen el proceso de acumulación bajo un discurso de objetividad que oculta, consciente $o$ inconscientemente por parte del investigador, la reproducción de la llamada corriente principal de la ciencia, son considerados como científicos con mayores posibilidades de ser publicados.

2. En las llamadas ciencias duras encontramos en las revistas científicas, escasos análisis críticos a los grandes avances mercantilizados en diversas materias, tal es el caso de la biotecnología, la producción de transgénicos y fármacos entre otros, esto es así a pesar de, como dice Lander (2006:46), la existencia de múltiples perspectivas críticas que develan la articulación entre la producción de conocimiento y el modo de producción capitalista y de la realización de "intensos debates y confrontaciones políticas". Las revistas de las ciencias duras en nuestra región reproducen el perfil político que exigen los índices llamados de prestigio internacional alineados a la corriente principal de la ciencia.

En el campo de la medicina está casi ignorada la medicina alternativa o naturista y se excluyen trabajos que interpelan los intereses mercantiles de la medicina, por ejemplo: el carácter mercantil de la fecha de vencimiento de los medicamentos, el papel directo de los productos de la naturaleza en la salud, la apropiación mercantil de los saberes ancestrales, la escasa promoción de la dimensión preventiva de la salud.

3. También excluyen en sus criterios, trabajos producto de metodologías de investigación alternativa: investigación-acción e investigación participativa, las cuales tienen como rasgos fundamentales la incorporación de sujetos no tradicionales como las comunidades y centran la atención en la transformación de la realidad cuestiones que se distancian de métodos, sujetos y fines de la corriente principal de la ciencia. Estas investigaciones no son consideradas científicas porque, a la luz de la lógica cientificista hegemónica, transgreden la autoridad del científico o experto considerado el único portador del conocimiento.

4. La sistematización de experiencias, tampoco es considerada conocimiento científico por lo tanto se excluye de las revistas. La sistematización de experiencias es definida por Jara (citado por Borjas, 2003:16) como "aquella interpretación crítica de una o varias experiencual intentan evadir su responsabilidad en la decisión, ocultando que la decisión de qué publicar y la escogencia de los árbitros es de quienes dirigen la revista. 
cias, que a partir de su ordenamiento y reconstrucción, descubre o explicita la lógica del proceso vivido, los factores que han intervenido en dicho proceso, cómo se han relacionado entre sí y porqué lo han hecho de ese modo... nos hace objetivar lo vivido, hacer un alto para tomar distancia de lo que hemos experimentado vivencialmente y convertir así la propia experiencia en objeto de estudio e interpretación teórica, a la vez que en objeto de transformación". A través de la sistematización de experiencias se produce conocimiento sobre la práctica concreta, con rigor científico, su exclusión revela desprecio por el pensamiento crítico, por la posibilidad de construir teoría a partir de nuestra realidad y por la investigación para transformar la realidad. Para Jara (2012), autor que constituye una referencia latinoamericana en materia de sistematización de experiencias, el reto es "construir nuevas epistemologías que se enfrentan a las formas tradicionales de producir conocimiento científico y a las formas dominantes de producción y circulación de saberes".

5. Los temas centrales, los cuales ocupan un espacio importante en las revistas, casi nunca consideran las realidades estudiadas por el pensamiento alternativo a la corriente principal. En el campo de las ciencias sociales se excluyen numerosos temas ${ }^{3}$ que solo eventualmente ocupan el centro en nuestras re- vistas. En definitiva los temas centrales son reproductores de la corriente principal. Eventualmente las revistas incorporan como temas centrales, temas locales relevantes para comprender la realidad latinoamericana, tales como la Amazonía, la participación comunitaria, los saberes ancestrales, pero tratados desde una óptica conservadora, con exclusión de posturas críticas que la confrontan. La incorporación de estos temas, que son muy estudiados por el pensamiento contrahegemónico, contribuye a legitimar las revistas como inclusivas de la diversidad de pensamiento pero finalmente como hemos dicho se hace con un tratamiento que tributa a la corriente principal de la ciencia.

6. Las fuentes de información contrahegemónicas son objetadas y se reclaman fuentes hegemónicas. Este tipo de actitud constituye una práctica autoritaria que obedece a la estructura jerarquizada conocimiento, la cual asigna a los sujetos y al conocimiento de la corriente principal una superioridad sobre el conocimiento contrahegemónico.

La invisibilización del conocimiento alternativo es parte de la estructura de poder de la organización científica. Se visibiliza de los llamados países periféricos aquellos conocimientos que aportan a la corriente principal, se excluye el conocimiento contrahegemónico a la corriente principal.

3 Feminismo, decolonialidad, salud colectiva, organización comunitaria, organizaciones emergentes, comunas, nacionalizaciones, recuperación empresarial por los trabajadores, organización del trabajo, pedagogía crítica, educación emancipadora, interculturalidad, entre muchos otros. 
Esta estructura de poder ha creado mecanismos que la favorecen, entre éstos se encuentra el control por parte del $\mathrm{SCl}$ del perfil de las revistas científicas, se imponen criterios a las revistas para ser incluidas en los índices, algunos de estos criterios son de exclusión sutil.

La creación de índices de acceso abierto ha permitido mejorar la obtención de información del llamado conocimiento periférico por parte de los usuarios, no obstante las condiciones de invisibilidad del conocimiento contrahegemónico se mantienen debido a que se imponen criterios similares al SCl para el ingreso.

La creación de revistas con pensamiento contrahegemónico ha sido muy lenta en América Latina. Ante la ausencia de apoyo de las revistas científicas al conocimiento alternativo, éste se encuentra actualmente a merced en el mejor de los casos, de estrategias de publicación con poca visibilidad y en el peor de los casos, reducido a debates en pequeños grupos con pocas posibilidades de reproducción.

Los repositorios institucionales que incluyen diversos documentos y no solo artículos publicados en revistas, han permitido que se publiquen trabajos con pensamiento alternativo y ha facilitado a los investigadores mayor literatura, muchas veces subestimada por la corriente principal. Esta estrategia de visibilidad del conocimiento ha sido una alternativa para investigadores, que han migrado a trabajar con corrientes alternativas a la llamada corriente principal. La invisibilización del conocimiento alternativo por parte de las revistas científicas, ha sido un freno a la disponibilidad de este conocimiento por parte de los investigadores.

\section{Las revistas científicas en Venezuela vs la nueva política pública de ciencia y tecnología en el país}

Las revistas científicas venezolanas se reprodujeron a raíz de una política pública de promoción de la investigación con la corriente principal en el contexto neoliberal a inicios de los años noventa a través del Programa de Promoción al Investigador (PPI), administrado por la Fundación Sistema de Promoción al Investigador (FSPI). Fue una política que tuvo cambios en 2002, producto del advenimiento de la Revolución Bolivariana, al incrementarse los índices reconocidos para calificar las revistas en las cuales publicaban los investigadores, es decir en este momento se amplió el criterio, pero conservando los criterios fundamentales de inserción en la corriente principal de la ciencia.

El centro de la evaluación lo constituía la acreditación de los investigadores por sus publicaciones en revistas registradas en el SCl. Al mismo tiempo el gobierno financia revistas utilizando los criterios de la corriente principal de la ciencia.

El referido modelo se mantuvo hasta 2010 cuando se suspendió el apoyo financiero del gobierno a las revistas y la acreditación a los investigadores por el $\mathrm{PPI}$, mientras que se diseñaban nuevas políticas.

Para algunos autores (Aguado y Becerril, 2016) Venezuela presenta una crisis de producción científica desde 2009, por la caída de trabajos registrados en SCOPUS. Evaluar la producción cien- 
tífica de un país desconociendo el contexto y asumiendo solo las publicaciones en revistas registradas en un índice de la corriente principal, es a nuestro juicio una forma de reproducir la actual estructura de poder de la organización de la ciencia, que Venezuela desde una postura emancipadora intenta subvertir.

Más allá de los registros de REDALYC y SCOPUS (por cierto varias revistas venezolanas de mucha trayectoria no están registradas en SCOPUS según el trabajo de Aguado y Becerril, 2016), la producción científica venezolana debe ser evaluada considerando el conocimiento contrahegemónico que se promueve y que tienen otros medios de visibilidad (repositorios institucionales, libros, memorias de eventos, entre otros).

Una fuente de información más cercana a la realidad de la producción científica la constituyen los repositorios institucionales y aún así quedaría mucha producción científica invisibilizada por cuanto las universidades y otras instituciones emergentes no han creado condiciones para registrar su producción científica.

La vieja política científica y tecnológica promovida por las élites científicas desde los años sesenta y fortalecida en la etapa neoliberal a través del Promoción al Investigador (PPI), sufre un quiebre con el advenimiento del gobierno bolivariano, "nos preguntamos ¿cuál es la mano invisible que impulsó este programa en varios países de la región?. No dudamos de la participación de los organismos multilaterales, con imposiciones a través de financiamiento, pero más allá de esto se encuentra una concepción neutral de la ciencia asumida por gran parte de la comunidad científica y por quienes han tenido la responsabilidad de dirigir este sector social y que se arropan con el manto de la denominada corriente principal de la ciencia, considerada válida para toda realidad" (Ochoa, 2011:114).

La nueva política comienza a formularse desde 1999 cuando se incorpora el tema en la Constitución de la República Bolivariana de Venezuela (CRBV) (ANC, 1999) y se crea el Ministerio de Ciencia y Tecnología (MCT). Desde entonces se han producido cambios importantes: 1) A través de numerosos instrumentos legales, entre los cuales destaca la Ley Orgánica de Ciencia, Tecnología e Innovación conocida como LOCTI (2001-2005) que entre otras cuestiones novedosas incorpora la obligación de las empresas a aportar a la producción científica, tecnológica y de innovación y 2) Desde varias instancias del aparato estatal, en especial MCT. Se trata de una política opuesta a la política científica hegemónica guiada por la reproducción de la corriente principal de la ciencia cuyo destino es el consumo de la comunidad científica internacional.

La nueva política científica se propone fundamentalmente, desde el MCT: 1) Producir conocimiento para transformar la realidad, atendiendo las necesidades acordes con los objetivos nacionales, por lo que adquiere especial importancia: la innovación, en el contexto de búsqueda de superación del modelo económico rentista petrolero, y la definición de áreas prioritarias alineadas a planes nacionales, 2) Descolonizar y democratizar la actividad científica, incorporando nuevos modos de producción de conocimiento y nuevos sujetos en especial a las comuni- 
dades, proceso que pasa por el diálogo de saberes, para el rescate de los saberes ancestrales y populares y la articulación de investigadores y usuarios del conocimiento, en cuyo contexto se han promovido las redes como estrategia organizativa. Según Santos (2010:53) el uso de prácticas científicas alternativas pasa por promover "la interacción e interdependencia entre conocimientos científicos y no científicos". En la ruta hacia los referidos propósitos se creó la Misión Ciencia, que constituye un macro-programa para impulsar el modelo contrahegemónico.

Como parte de la nueva política científica, para las universidades emergentes, creadas por la Revolución Bolivariana ${ }^{4}$, el gobierno define métodos para promover la articulación de las funciones sustantivas de dichas universidades y la participación de las comunidades a través de una unidad curricular denominada proyecto, lo cual crea condiciones para la producción de conocimiento alternativo.

Intentando avanzar en la nueva política se cierra el PPI y se abre el Programa de Estímulo a la Innovación y a la Investigación (PEII) incorporando nuevos criterios que valoran: 1) El conocimiento producido cuyo destino no son las publicaciones indizadas, sino que se pone especial atención en las revistas nacionales, 2) El trabajo en grupo, considerando la participación en redes, 3) La articulación científica con las comunidades 4) La formación de talento por parte de los investigadores, 5) Los desarrollos tecnoló- gicos incluyendo la tecnología producida por sujetos no académicos con potencial para sustituir importaciones y dirigida a pequeños productores, 6) La investigación programada y 7) Muy especialmente la articulación de la investigación con las áreas prioritarias en ciencia, tecnología e innovación (ONCTI, 2011). Se trata de un programa de promoción de la investigación que rompe con la lógica del PPI para avanzar hacia una nueva práctica científica en el país.

En la misma línea de transitar por una ruta hacia la transformación de la investigación en el país, también en 2011 el MCT ofrece financiamiento para la investigación en áreas articuladas al Plan Nacional que promueven la producción de conocimiento alternativo. En dicha oferta se definen ocho áreas de investigación y a lo interno de cada área se definen subáreas y líneas de investigación, producto de operacionalización del Plan Nacional Simón Bolívar (2007-2013). Las áreas se definen por su aporte a la transformación de la realidad con sub-areas que constituyen temas alternativos. Estas ofertas tienen lugar a través convocatorias electrónicas para el financiamiento cuyos formatos exigen al aspirante escoger áreas, subáreas y líneas de investigación.

En síntesis, asistimos desde 1999 a una política pública de producción de conocimiento alternativo, al menos formalmente, que en la práctica se ha visto disminuida por obstáculos burocráticos, pero que de alguna manera ha permitido 
incrementar la "Ciencia Nuestra", como la denomina Vargas (2012), que siempre ha tenido presencia en nuestros centros de investigación pero que su difusión se ha visto afectada por restricciones de las revistas científicas del país, en su mayoría alineadas con la llamada corriente principal de la ciencia.

La política de difusión del conocimiento no ha tenido la misma dinámica de transformación, ha sido más lenta, no obstante ha habido esfuerzos importantes desde el Ministerio de la Cultura como veremos más adelante.

Desde el Ministerio del Poder Popular para Educación Universitaria, Ciencia y Tecnología (MCTI) la política de difusión fortalecida en los noventa, que valora profundamente las publicaciones de impacto en los índices controlados por el pensamiento hegemónico, tuvo continuidad en la evaluación del PPI por Observatorio Nacional de Ciencia, Tecnología e Innovación (ONCTI) con cambios incrementales que no daban un salto hacia la difusión del conocimiento alternativo. Por una parte se siguieron privilegiando las revistas indizadas en el $\mathrm{SCl}$, con algunos cambios incrementales al valorar las revistas acreditadas por índices latinoamericanos que en esencia se encontraban alineados al $\mathrm{SCl}$, además de los mencionados en páginas anteriores:
SCIELO y REDALYC, se acreditan revistas indizadas en el índice venezolano: Registro Venezolano de Publicaciones Científicas y Tecnológicas (REVENCYT), el cual ha tenido muy poco impacto en la región, y algunos directorios mexicanos (CLASE, LATINDEX). Las revistas del país se vieron favorecidas en su reconocimiento por la posibilidad de ingreso a estos índices latinoamericanos, muy pocas revistas del país se encuentran en el SCl.

También desde el MCTI, concretamente en el Fondo Nacional de Ciencia Tecnología e Innovación (FONACIT) se realizaron cambios en la política de acreditación para el financiamiento a las revistas científicas del país, que igualmente calificamos de incrementales, tales como: creación de nuevos criterios de visibilidad de las revistas ${ }^{5}$ que consideraron la distribución, el origen de los trabajos y el arbitraje.

Fueron cambios que no lesionaron la dinámica de las revistas del país desde el punto de vista de su lógica favorecedora de la difusión del conocimiento hegemónico. Excepción de esta lógica lo constituye la Revista Diálogo de Saberes editada desde el 2008 por la Universidad Bolivariana de Venezuela, creada en 2003 como parte de las universidades emergentes.

La búsqueda de ruptura a esta lógica pasó por la suspensión de la política

5 En la distribución los indicadores de visibilidad fueron: canje con otras revistas e instituciones nacionales, canje con revistas e instituciones extranjeras, Donación a instituciones, Venta por suscripción y número suelto. De acuerdo al origen de los trabajos se consideró: autores de otras instituciones nacionales, autores de otras instituciones extranjeras. En el arbitraje fue valorada la inclusión de árbitros de otras instituciones nacionales y árbitros de instituciones extranjeras (FONACIT, 2008). 
de financiamiento a las revistas y un lapso de ambigüedad que aún no se ha resuelto, lo que demuestra que el gobierno se encuentra en deuda con la búsqueda de alternativas que promuevan transformaciones en las revistas editadas en el país.

En este contexto el gobierno apoya desde 2012 el llamado Índice Venezolano de Publicaciones Periódicas "ClaCalia" con nuevos criterios ${ }^{6}$ para dar visibilidad al conocimiento alternativo. Según Rodríguez (2016) el índice es producto de un proyecto PEIl financiado por FONACIT que luego es asumido por la institución, para lo cual subcontrata una ONG (Ejercito Comunicacional de Liberación). No obstante el funcionamiento de este índice no está claro, en el 2012 con los criterios de este índice se realizaron evaluaciones a las revistas para conocer la presencia de pensamiento contrahegemónico.

La promoción de revistas que promuevan la difusión del conocimiento alternativo por el ministerio es un tema que sigue pendiente. Por el contrario, en los actuales momentos estamos en presen- cia de una evaluación del sistema de acreditación de las revistas, esta vez desde el ONCTI que es la institución que administra el PEI, lamentablemente se están retomando los criterios de reproducción de la corriente principal de la ciencia.

Desde el 2014 el ONCTI trabaja en un proyecto de integración con la Universidad de Los Andes (ULA) para impulsar el índice REVENCYT asumiendo éste la acreditación de las revistas venezolanas y la posibilidad de financiamiento de las revistas acreditadas por ONCTI. Si bien la estrategia está en proceso de formulación, la orientación asumida por el ONCTI en conjunto con los Servicios Bibliotecarios de la Universidad de los Andes (SERBIULA) es promover el conocimiento hegemónico en las revistas latinoamericanas ${ }^{7}$, los avances en la ejecución identificados hasta ahora consisten en la formación de editores de las revistas venezolanas para la acreditación en índices de la corriente principal (SCI, REDALYC, SCOPUS y SCIELO), la cual es provista por el REVENCYT perteneciente a la ULA.

6 Innovación científica, Tecnológica, metodológica y/o teórica; contextualización; cuestionamiento al sistema de dominación capitalista; promoción de la resistencia a la depredación ecológica; generación de intercambios y redes de promoción de conocimiento a nivel local, nacional, latinoamericano y/o caribeño; uso de licencias de libre acceso de conocimientos de contenidos; promoción de la transdisciplinariedad, la interacción y trasformación recíproca entre disciplinas; incorporación de métodos de investigación cualitativa; articulación del conocimiento con saberes, tradiciones y culturas locales; aportes a la unidad latinoamericana y caribeña, para la solidaridad con los pueblos del Sur para el desarrollo de la multipolaridad y la compresión de los problemas comunes de la humanidad; contribución a la superación de la «colonialidad del saber»; gratuidad o precio accesible de la publicación; entre otros (CLaCalia, 2012).

7 En las actividades formativas en las que participó REVENCYT, producto de la alianza del ONCTI y la ULA, sus organizadores manifestaron que "preparar a los editores de las revistas científicas venezolanas es ir pensando en las publicaciones de las corrientes principales, tales como, Science Citation Index, Redalyc, Scopus y Scielo" (Sánchez, 2014). 
Paralelo a la casi ausencia de incorporación del pensamiento contrahegemónico por la revistas científicas, el gobierno desde el Ministerio de Cultura ha promovido este pensamiento. Por una parte con la democratización del libro a través de la Editorial El Perro y la Rana, que edita pensamiento crítico y promueve la lectura con la venta simbólica de los libros en sus Librerías del Sur. Por otra parte el gobierno bolivariano ha promovido el conocimiento crítico con el Premio Libertador creado en 2005 al cual se han presentado reconocidos investigadores de América Latina, con temas que tributan a una nueva estructura de poder de la ciencia en la región. En la $X$ edición del 2015 se presentaron 55 libros de autores de 16 países, el premio incluye la publicación del libro por el gobierno y su distribución gratuita en gran medida.

\section{Conclusiones}

En el país se ha avanzado en la producción de conocimiento alternativo a la corriente principal de la ciencia desde fines de los noventa con el advenimiento de una política científica y tecnológica alternativa promovida desde el MCTI.

Dicho conocimiento tiene restricciones a través de diversas estrategias, para ser publicado en las revistas científicas las cuales se encuentran alineadas a la estructura dominante de las publicaciones, apegadas a los criterios de índices promotores de la llamada corriente principal de la ciencia, los cuales se han incrementado con el surgimiento de índices latinoamericanos que asumen tales criterios, entre los cuales se encuentra un índice venezolano aunque de poco impacto.

La existencia de repositorios en muchas universidades en los cuales se publican numerosos productos distintos a los artículos (ponencias en eventos, trabajos de tesis, ascenso, entre otros) ha permitido cierta visibilidad de pensamiento contrahegemónico, estrategia que se ha visto restringida por inexistencia de repositorios de acceso libre en las universidades emergentes, donde se hacen esfuerzos por superar los paradigmas tradicionales de investigación.

La política de difusión del MCTI no ha aportado a la transformación del perfil de publicaciones de las revistas científicas, los cambios realizados a dicha política han sido incrementales hasta el 2010 cuando hace crisis la política suspendiéndose el registro de las revistas y el financiamiento.

EI MCT está llamado a retomar la política de financiamiento a las revistas venezolanas, acompañada de creación de políticas de difusión del conocimiento alternativo por parte de dichas revistas. Existen ideas sobre criterios de evaluación alternativos en la experiencia de ClaCalia, los cuales se encuentran disponibles como insumo para la evaluación del sistema de acreditación de revistas en el país.

La reciente celebración de alianzas -en proceso- desde el ONCTI con el índice nacional (REVENCYT) para retomar la política de acreditación y financiamiento de revistas científicas ha transcurrido sin la interpelación de los criterios de evaluación de las revistas aplicado por dicho índice lo cual significa la legitimación de la 
corriente principal de la ciencia en las revistas científicas nacionales y el consecuente bloqueo de la investigación alternativa.

Asistimos de esta forma a la existencia en el MCTI de una contradicción entre la política pública de producción de conocimiento contrahegemónico y la política de difusión con cambios incrementales que no impactan la transformación y en puerta proyectos que reproducen la vieja política.

No obstante desde el Ministerio de Cultura se ha promovido la publicación de pensamiento crítico a través de diversas vías, en particular es relevante la publicación de libros y la promoción de producción de pensamiento crítico a través del Premio Libertador. Es una política que ha dado visibilidad a la producción científica contrahgemónica del país.

\section{Referencias bibliográficas}

\section{Aguado, Eduardo y Becerril, Arianna (2016).} Producción científica venezolana: Apuntes sobre su pérdida de liderazgo en la región latinoamericana. $\mathbf{R e}$ vista Venezolana de Gerencia (RVG). Año 21, No. 73, Facultad de Ciencias Económicas y Sociales de la Universidad del Zulia (LUZ). Maracaibo. Venezuela.

Asamblea Nacional (AN) (2013). Plan Nacional 2013-2019. Caracas.

Asamblea Nacional Constituyente (ANC) (1999). Constitución de la República Bolivariana de Venezuela (CRBV). Caracas.

Borjas, Beatriz (2003). Metodología para sistematizar prácticas educativas: Por las ciudades de Italo Calvino. Federación Internacional de Fe y Alegría. Caracas.
Boscán, Elizabeth (2016). Reflexiones teóricas sobe la Gestión de la política científica y tecnológica. Avances de la tesis doctoral. Maracaibo. Venezuela.

FONACIT (2008). Planilla de Evaluación Integral de Revistas. MCT-FONACIT. Caracas.

Guedón, Jean-Claude (2011). El acceso abierto y la división entre ciencia "principal" y "periférica". Revista C y E. Año III, $\mathrm{N}^{\circ}$, Consejo Latinoamericano de Ciencias Sociales (CLACSO). Buenos Aires.

Jara, Oscar (2012). El desafío político de aprender de nuestras prácticas. CEP Centro de Estudios y Publicaciones Alforja. Costa Rica.

Lander, Edgardo (2000). ¿Conocimiento para qué? ¿Conocimiento para quién? Reflexiones sobre la geopolítica de los saberes hegemónicos. Revista Venezolana de Economía y Ciencias Sociales. Vol. 6 No. 2, Universidad Central de Venezuela. Caracas.

Lander, Edgardo (2006). La ciencia neoliberal. En: Los desafíos de las emancipaciones en un contexto militarizado. Consejo Latinoamericano de Ciencias Sociales (CLACSO). Buenos Aires.

Lander, Edgardo (2009). Tendencias dominantes de nuestra época ¿se nos agota el tiempo? Revista de Investigación Científica Compendium. No. 22, Universidad Centroccidental Lisandro Alvarado. Barquisimeto, Venezuela.

Observatorio Nacional de Ciencia, Tecnología e Innovación (ONCTI) (2011). Reglamento del Programa de Estímulo a la Investigación (PEI). Caracas.

Ochoa Henríquez, Haydée (2011). Del PPI al PEI: hacia el avance de la nueva política científica y tecnológica del gobierno bolivariano. EnI@ce. Revista Venezolana de información, tecnología y conocimiento. Año 8 No. 2, 
Universidad del Zulia. Maracaibo. Venezuela.

Presidencia de la República (2007). Proyecto Nacional Simón Bolívar - Primer Plan Socialista (PPS). Caracas.

Santos, Boaventura de Sousa (2010). Descolonizar el saber, reinventar el poder. Ediciones Trilce-Extensión Universitaria. Universidad de la República. Montevideo.

Sánchez, Ramiro (2014). Culminó con éxito taller para Editores de Revistas Científicas. Portal del Vicerrectorado Acadé- mico de la ULA. Disponible en: http:// www2.ula.ve/viceacademico. Consultado el 1-10-2016.

Rodríguez, Lennymar (2012). Investigadora del IVIC. Entrevista realizada el 20 de noviembre de 2014. Caracas.

Varsavsky, Oscar (1969). Ciencia, política y cientificismo. Centro Editor de América Latina. Buenos Aires.

Varsavsky, Oscar (2006). Hacia una política científica nacional. MCT-FONACIT. Caracas. 